\title{
An Ontology to Model e-portfolio and Social Relationship in Web 2.0 Informal Learning Environments
}

\author{
D. Taibi, M. Gentile, G. Fulantelli, M. Allegra
}

\author{
Davide Taibi, Manuel Gentile, Giovanni Fulantelli, Mario Allegra \\ Italian National Research Council \\ Institute for Educational Technologies \\ Via Ugo La Malfa 153 \\ 90146 Palermo, Italy \\ E-mail: \{davide.taibi,manuel.gentile,giovanni.fulantelli,mario.allegra\}@itd.cnr.it
}

\begin{abstract}
Web 2.0 applications and the increasingly use of social networks have been creating new informal learning opportunities. Students interact and collaborate using new learning environments which are structurally different from traditional e-learning environments. In these informal unstructured learning contexts the boundaries between the learning contexts and social spheres disappear, and the definition of the students competences appears more and more important. In this paper we propose a semantic web approach in order to create the basis for a software platform to model learner profiles.

In particular we propose to extend the FOAF ontology, used to describe people and their personal relationships, with an ontology related to the IMS Learning Portfolio used to model students' competencies. This ontology could be a fundamental layer for a new Web 2.0 learning environment in which students' informal learning activities carried out in social networks can be managed and evaluated.
\end{abstract}

Keywords: semantic web, e-portfolio, social networks, informal learning.

\section{Informal learning and social communities}

The significant changes in society that Castells in [1] sums up in what he calls "The Rise of the network society" also have considerable implications in the definition of learning activities. The Information Society dramatically increases the opportunities for knowledge acquisition. Beyond the structured training activities designed by specialists in the education field, we have to consider the large number of educational opportunities related to everyday activities that define the so-called "informal learning" [2]. In this perspective, the concept of networked learning is drastically changing. The informal learning opportunities created by information technologies, such as Web 2.0 applications and social networks, allow users to interact and collaborate in new ways thus leading to the definition of new learning environments; these are structurally different from traditional e-learning environments, since the boundaries between the learning contexts and other social spaces tend to disappear. In these unstructured learning contexts, the definition of the skills acquired by the users is a central objective. Consequently, the use of software environments that model learner profiles and can deal with them in a semantic way appears increasingly important.

In [3] the authors argue that learning is related to the activities, the environmental and cultural contexts in which it is developed and therefore social interaction is a critical factor. From this point of view learning can be described as a process: students are involved in a community of practice that represents knowledge and behavior in which students play a more active role in the cultural sphere. The concept of situated learning comes from Vygotsky's social development theory, which affirms that social interaction has a fundamental role in the knowledge development process [4].

This theory argues that situated learning is generally unintentional and for this reason learning is more effective if the student is a member of a community of practice he has chosen to join rather than 
being assigned to a group by external actors such as teachers. The social aspect in learning activities is extremely important and leads to a further consideration. For example, Tinto claims that participation in a collaborative learning group allows students to develop a supporting network, that helps students to maintain relations with a wider social community [5].

A peer-to-peer community promotes participation in learning activities. Moreover, the communities of learners provide students with the opportunity to satisfy simultaneously both social and academic requirements. These unstructured learning contexts give rise to the need to measure and asses the acquired knowledge; the traditional competence based certification systems are not designed for this type of environment and for this reason are less suitable in this kind of educational context. The semantic web provides a technological substrate which can overcome the limits of current web technologies, setting the base for creating ontological systems in order to model competences in informal educational contexts that are being developed in web 2.0 environments.

In this paper we consider the problems connected to the description of competences in informal learning environments within social networks mediated by technologies. In particular, we propose the integration of the FOAF (Friend Of A Friend) ontology, which is used to model people and their personal contacts, with semantic ontology related to student e-portfolios used to model their competences. The use of ontologies and the surrounding semantic web technologies allow us to create relationships between the students ongoing educational experiences and the evolution of their social network. For this to happen, we integrate FOAF ontology with the IMS Learning Portfolio model in order to support the creation of a new Web 2.0 learning environment based on social networks and competences.

\section{Social Semantic Web}

At present a huge amount of shared contents such as bookmarks, images, videos and photos are being created within so called web 2.0 applications, very popular in social and personal spheres as well as in professional and organizational ones. They possess common features like the creation and sharing of contents (images, photos, papers), discussions (comments) and connections between users (group of friends, private messages, and so on). This scenario raises new considerations related to the sharing of social contributions between software applications and the interoperability of social networks.

Due to the heterogeneity of the nature of social contribution, sharing, searching, connecting and retrieving these kinds of contents has become more complex. The semantic web technologies provide standards and models which are useful for creating a network of data, with unified models which can represent data from different sources appropriately. The unification of semantic web technologies and social paradigms gives rise to "Social Semantic Information Spaces" in which information is socially created and managed, as well as being interconnected and available in a machine understandable format, promoting new methodologies to discover information present on the web [6].

Moreover, the semantic web offers a generic infrastructure to interchange, integrate and reuse structural data, in order to overcome the limits of Web 2.0 platforms. Currently, in fact, web 2.0 applications have search mechanisms based mainly on tags and few keywords. Adding semantics to the web would enable this kind of problem to be solved, by providing easier search mechanisms, supporting the reuse of contents and creating more connections between different types of contents. Moreover, the use of ontologies is useful to structure and elaborate information. Ontologies represents entity-relationship models related to a specific knowledge or practice domain. A typical web ontology contains the definition of classes, objects and their relationships, and a set of deduction rules that give inferential power about the concepts.

Through ontologies the semantic web provides the basis for enriching the resources description with a well defined meaning and in a comprehensible format which can be elaborated by software applications.

The strict relationship between documents produced in web 2.0 environments and the specific social network [7] bring us to consider the information objects as the result of the activities of the network; 
consequently, we should also represent social relationships in a well structured way, using approaches based on the semantic web concepts.

FOAF, the acronym of Friend of a friend, uses semantic web technologies, in particular the Resource Description Framework (RDF) and the Ontology Web Language (OWL), to define a machine-readable ontology describing people, their activities and their relations to other people and objects. FOAF is useful for describing social networks and their relationships.

\section{Learner model in social semantic networks}

Educational activities adopt web 2.0 technologies and social networks more and more frequently. The interaction paradigms at the basis of web 2.0 technologies and social networks are different from those adopted by conventional e-learning tools.

FOAF is considered the most common vocabulary for constructing social networks, it has been very successful in the applications that use semantic web technologies, and it is useful to model learner in social networks [8].

Each student can be described through an FOAF file that can be extended and modified at any time. This is useful, for example, for publishing data regarding students using the URI that represent them. To facilitate the creation of the profiles it is possible to use an interface based on foaf-a-matic; in this way, it will be possible to describe social links in the community of practice. Defining ontologies it is possible to use a inferential engine like Jena and the SPARQL language to work with data and generate new knowledge about the domain. As reported by [9] there are several advantages in using the FOAF approach to model student profiles:

- the use of RDF facilitates extensibility and interoperability

- the presence of different extensions of the FOAF vocabulary, makes FOAF very flexible

- the creation of FOAF files is simplified by the use of foaf-a-matic

- FOAF simplifies the identification of people with common interests, which is essential for creating communities of practice

To use FOAF in a learning context, it is necessary to extend this model to include specific characteristics related to learning. Regarding this aspect we should take into consideration elements related to: the extension of the FOAF vocabulary to include specific information regarding students activities; the consideration of privacy problems in sharing personal information; the evaluation of the ties strength between students belonging to the group.

In conclusion, using FOAF as a basis for learning models makes it possible to exploit the benefits of the numerous existing tools, and also to use the extension of the model to define specific aspects and personal and group relationships, which are indispensable for creating and supporting social learning networks.

FOAF is used successfully to describe a student profile, in particular the profile can be extended to bring together information coming from other models containing student data, like, for example the competences described following the IMS e-portfolio standard. The model of students competences plays a key role in making the use of social networks to better support learning activities.

\section{4 e-portfolio and ontologies in social learning environments}

An e-portfolio is defined by the EDUCAUSE NLII (National Learning Infrastructure Initiative) as " $a$ collection of authentic and diverse evidence, drawn from a larger archive, that represents what a person 
or organization has learned over time, on which the person or organization has reflected, designed for presentation to one or more audiences for a particular rhetorical purpose."

As sustained by [10], an e-portfolio can be used to developmental, presentation, assessment purposes, and it can contain different information related to personal and professional achievements, competences, digital works. This relevant information about students can be stored and maintained by different institutions in different sites, so the management can be improved by the use of web-based e-portfolios. A key concept in this scenario is the interoperability between different institutional systems which requires a unified model describing students e-portfolios.

The pedagogical objectives of e-portfolios are various: they allow students to describe their learning path, increase awareness of their strengths and weaknesses, take responsibility and increase their autonomy and have a unified way of presenting their competences.

At present, the lack of common standards to describe e-portfolio information means that most eportfolio systems are using different proprietary formats to store this type of information, and moreover, they don't provide features for importing and exporting e-portfolio information from other systems. In this scenario the interoperability between e-portfolio systems is hindered, and for example, it is difficult to integrate the e-portfolio information coming from a university system and from an enterprise.

For these reasons it is desirable to use a common standard in order to unify the description processes of competences in lifelong learning.

There are two main standards for describing student learning experiences. The IEEE Learner Model working group has defined the Public and Private Information for Learners [11] as a standard for a student model, with the aim of gathering information related to competences, personal data, learning style, and so on. This standard considers six types of data related to Personal, Relations, Security, Preference, Performance and Portfolio information; in addition, it is possible to extend and integrate the standard in order to enrich the student description.

In 2005 the IMS consortium released the IMS ePortfolio Practice and Implementation Guide [12]. This specification uses the XML language to define the characteristics of an e-portfolio. XML is at the basis of the semantic web layer cake, so this specification constitutes the first step towards a semantic description of student competences. The use of specific ontologies can enrich this description by considering also the relationships between the concepts that are at the basis of e-portfolio systems.

For example, figure 1 shows the e-Portfolio Activity concept, its properties and its relations reported in our ontology.

An e-Portfolio can bring together different kinds of information such as: digital and non digital works; activities in which the student has participated, is participating, or plans to participate; competences and skills of the student; students achievements, whether or not certificated; student's preferences; student's goals and plans; student's interests and values; any notes, reflections or assessments relevant to any other part; the results of any test or examination taken by the student; contextual information to help the interpretation of any results.

\section{Semantic framework for e-portfolio management}

Many educational approaches are based on groupwork, since peer learning promotes cognitive processes. There are many different kinds of collaborative work that allow students to learn in different modalities, such as group discussions, group problem solving and group study. The form of collaboration differs according to the duration, the complexity and the level of collaboration.

Social interactions can help students to share their experiences and to work collaboratively on relevant topics. In this sense social networks occupy a key role in the learning dynamics. The number of informal learning activities which take place in technology supported social networks is constantly increasing. Collaborative group activities are frequently used by teachers in the educational curriculum. In 


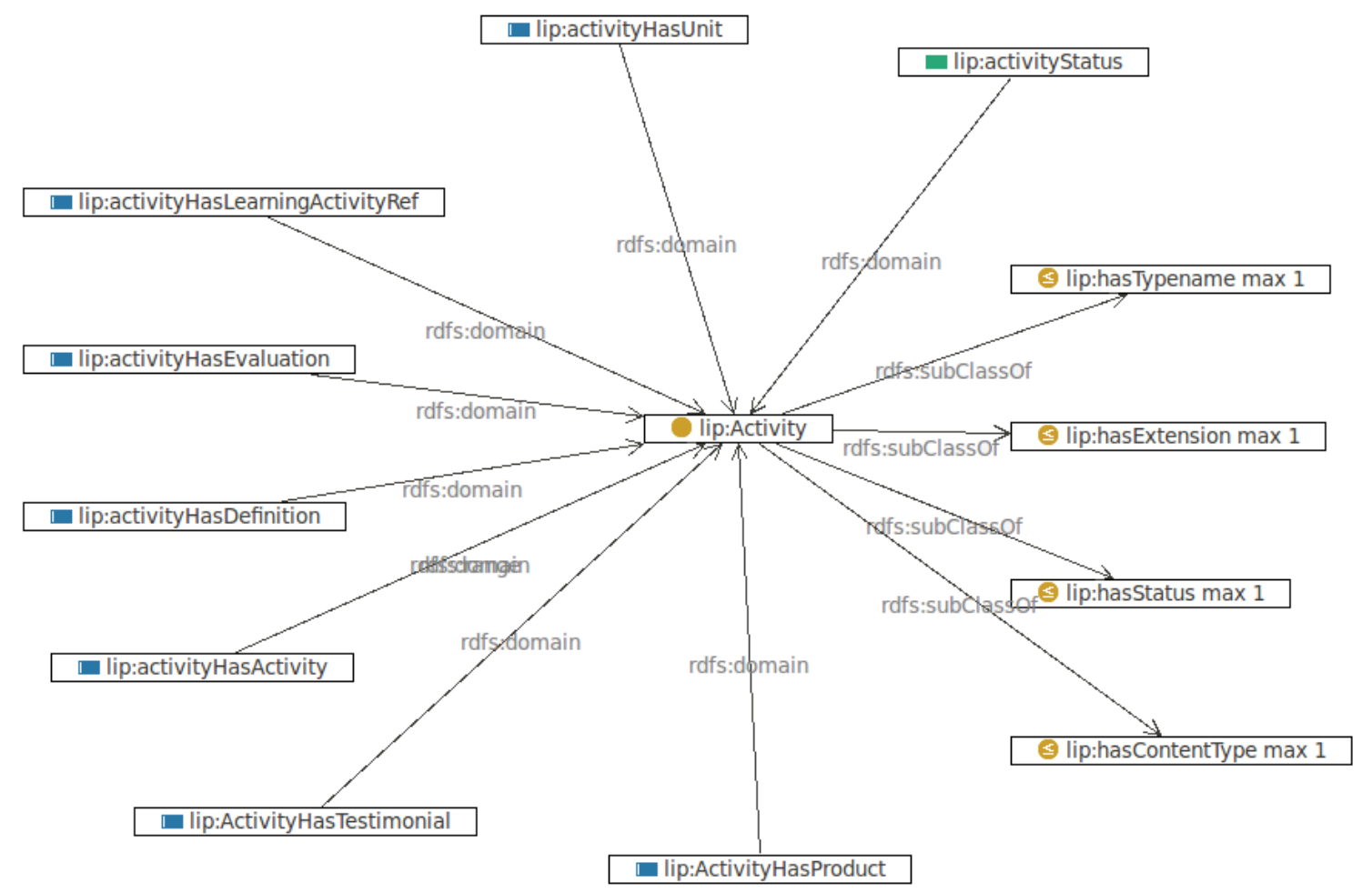

Figure 1: The activity Concept of the e-Portfolio Ontology

these activities it is necessary to create well balanced groups with the aim of maximizing the attainment of the learning objectives.

To ensure the success of a learning activity, teachers must consider the constraints that can affect the entire group or an individual performance, such as previous experiences by students in similar educational contexts, cultural background or interests and competences.

The importance of a system based on competences in informal learning environments such as those developed using social software is undeniable. For example, there are clear benefits in involving members with different levels of experience within the group in order to improve the dynamics of collaborative work in problem solving activities.

From this point of view it is increasingly important to have software applications that can store data related to the user profiles and process these data semantically.

The approach proposed in this paper consists in integrating and extending FOAF ontology, used for modeling contacts and personal relationship, with semantic data related to students competences.

Enriching the description of social networks using semantics can provide precious support for a more effective use of the network for educational purposes. Social learning experiences must consider the competences and the e-portfolio of the participants, so web semantic technologies are an essential substrate for merging models related to the social network description with models used to define and structure competences.

In particular, the use of ontologies and semantic web technologies makes it possible to relate the evolution of educational activities experienced by the students with their relationships.

The description of a students social network using FOAF, integrated with the definition of competences by means of the IMS model, is the basis for the creation of a competence based ontological system for virtual learning environments using social networks and web 2.0 technologies. The result is a learning environment which is no longer based on the transmission of information from teacher to student but rather is focused on the ability of the students to play an active role in their learning activities. 


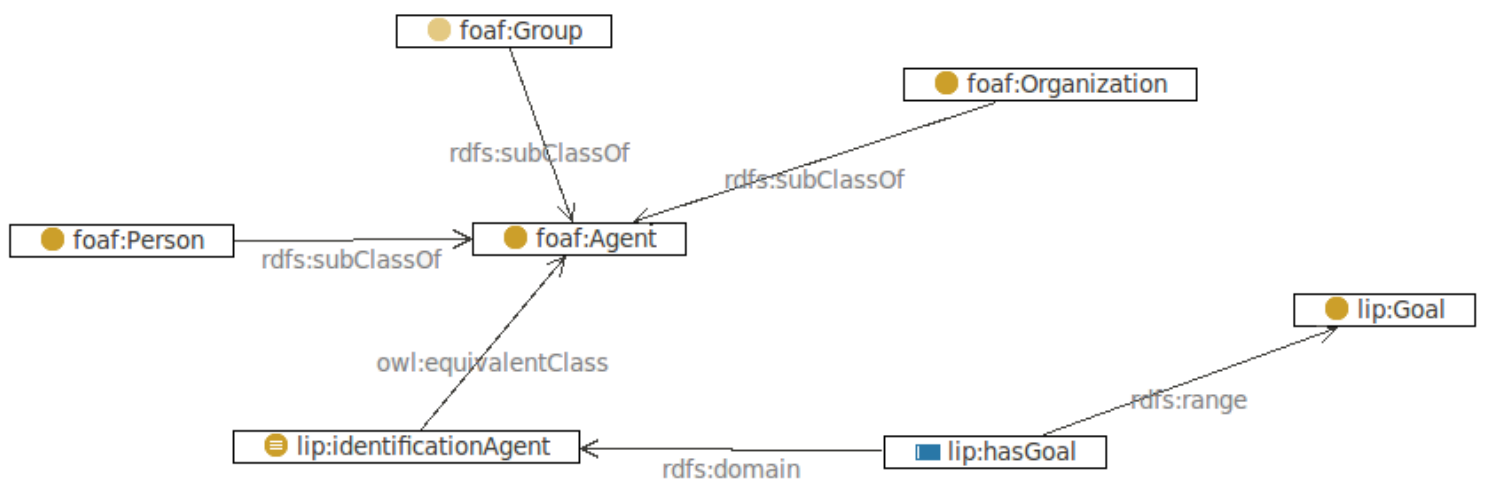

Figure 2: An example of the relation between FOAF and e-Portfolio in the proposed ontology

The figure 2 shows an example of how our ontology describes the relation between a concept of the e-Portfolio and a concept in FOAF.

As an example, we consider how our approach can be used to increase learning effectivness in informal learning activities that take place within an on-line social network environment. Using the ontology proposed in our work, it is possibile to describe both social relationships between students and the eportfolio for each student. By social relationships, we mean the friendship links explicitly declared by students within the on-line social network software, while the e-portfolio allows us to describe the competences acquired by students, their learning goals and so on. All this information can be available in a software platform that uses our ontology to:

- create a sub-group from a student's friendship group, which includes the friends that have common learning interests and objectives

- suggest new friends to a student by selecting the people from within the on-line social network who have specific competences in their portfolios that can help the student to achieve his learning objectives

In a social network it is important not only to be connected to other people but to be connected to the right people, depending on your goals. This is true not only for business or work experiences but it is also important for informal learning activities that take place in an on-line social network. The results of learning activities are highly influenced by the group in which students participate. Several studies have been conducted analizyng the impact of positive interdependence on the effectiveness of cooperation.

As stated by Johnson and Johnson one of the essential elements for efficacy in cooperation is positive interdependence [13]. The authors affirm that positive interdependence is structured in three categories: outcome, boundary and means. Our approach provides helpful conditions to support the first two categories of positive interdependence. In particular, the outcome categories include goals and rewards; in our case we create a sub-group of friends in which learning objectives and competences coincide, thus facilitating a structuring positive outcome interdependence in order to increase achievement and productivity [14], [15], [16].

Johnson and Johnson also state that "the boundary category includes: outside enemy (or negative interdependence with another group), identity (which binds members together as an entity), and environmental (such as a specific work area) interdependence" [13]. From this point of view, creating the sub-group from the existing friendship network increases some factors of boundary interdependence.

In conclusion, our ontological approach is useful for supporting the creation of learning groups, promoting positive interdependence that has a positive influence to produce higher achievement and 
productivity more then the membership in and of itself [17] and the interpersonal interaction itself [18], [19].

\section{Conclusions}

Collaborative group activities are frequently used by teachers in the educational curriculum. In these activities it is necessary to create well balanced groups with the aim of maximizing the attainment of the learning objectives.

To ensure the success of a learning activity, teachers must consider the constraints that can affect the entire group or an individual performance, such as previous experiences by students in similar educational contexts, cultural background or interests. The greater the number of constraints to consider, the more complex becomes the management of the learning experience.

Semantic web technologies offer the substrate needed to overcome the problems of social network with large groups of students. The versatility of these technologies means that they can be successfully applied for describing social networks and competences in learning experiences.

An interesting approach for creating an ontological system based on semantic web technologies that makes it possible to define a social network considering the competences of participants, the quality of the group and its robustness, is based on the use of an ontology as a result of an extension of the FOAF vocabulary, to create a semantic data base including specific references to educational paths.

In particular, the approach proposed in this work is based on the creation of a specifically designed ontology that extends FOAF ontology, in order to describe the domain of competences as defined by the IMS e-portoflio standard.

\section{Bibliography}

[1] M. Castells, The Information Age, Economy, Society and Culture Volume I: The Rise of Network Society, Blackwell, Oxford 1996.

[2] A. Andreatos, Virtual Communities and their Importance for Informal Learning International Journal of Computers, Communications \& Control, 2(1):39-47, 2007.

[3] J. Lave and E. Wenger, Situated Learning: Legitimate Peripheral Participation, Cambridge University Press, 1991.

[4] L.S. Vigotsky, Mind in Society. Harvard University Press. Cambridge, 1978.

[5] V. Tinto, Classrooms as communities: Exploring the educational character of student persistence, Journal of Higher Education, 68(6):599-622, 1997.

[6] J.G. Breslin, Social Semantic Information Spaces. In S.R. Kruk and B. McDaniel, Semantic Digital Libraries. Springer, 2008.

[7] J. Jung, J. Euzenat, Towards Semantic Social Networks. In Proceedings of the 4th European Semantic Web Conference, Innsbruck, Austria, pp. 267-280, 2007.

[8] A. Ounnas, I. Liccardi, H.C. Davis, D.E. Millard and S.A. White Towards a Semantic Modeling of Learners for Social Networks. In Proceedings of the International Workshop on Applications of Semantic Web Technologies for E-Learning (SW-EL) at the AH2006 Conference, Dublin, Ireland, 2006. 
[9] I. Liccardi, A. Ounnas, R. Pau, E. Massey, P. Kinnunen, S. Lewthwaite, M. Midy, C. Sarkar, The role of social networks in students' learning experiences. SIGCSE Bulletin 39(4):224-237, 2007.

[10] R. Mason, C. Pegler and M. Weller, E-portfolios: An assessment tool for online courses. British Journal of Educational Technology, 35,6, pp. 717-727, 2004.

[11] IEEE P1484.2.1/D8 : Draft Standard for Learning Technology - Public and Private Information (PAPI) for Learners (PAPI Learner) Core Features Sponsored by the Learning Technology Standards Committee of the IEEE Computer Society, 2001.

[12] IMS ePortfolio Practice and Implementation Guide, IMS Global Learning Consortium, 2005.

[13] D. W. Johnson, R. T. Johnson. An educational psychology success story: social interdependence theory and cooperative learning. Educational Researcher, 38(5), 365-379, 2009.

[14] M. Jensen, Cooperative quizzes in the anatomy and physiology laboratory: A description and evaluation. Advances in Physiology Education, 16(1), S48-S54, (1996).

[15] M. Jensen, D.W.Johnson, R. Johnson, Impact of positive interdependence during electronic quizzes on discourse and achievement. Journal of Educational Research, 95, 161-166, (2002).

[16] T. Matsui, T. Kakuyama, M. Onglatco, Effects of goals and feedback on performance in groups. Journal of Applied Psychology, 72, 407-415, (1987).

[17] N. Hwong, A. Caswell, D.W. Johnson, R. Johnson, Effects of cooperative and individualistic learning on prospective elementary teachers' music achievement and attitudes. Journal of Social Psychology, 133, 53-64, (1993).

[18] D. Mesch, M. Lew, D.W. Johnson, R. Johnson, Isolated teenagers, cooperative learning and the training of social skills. Journal of Psychology, 120, 323-334, (1986).

[19] D. Mesch, D.W. Johnson, R. Johnson, Impact of positive interdependence and academic group contingencies on achievement. Journal of Social Psychology, 128, 345-352, (1988). 